\title{
Clinical results of conservative management in patients with full-thickness rotator cuff tear: a meta-analysis
}

\author{
Masaki Karasuyama ${ }^{1}$, Masafumi Gotoh ${ }^{2}$, Keiji Tahara ${ }^{3}$, Junichi Kawakami ${ }^{1,4}, K^{2}$ Kazua Madokoro ${ }^{5}$, \\ Takashi Nagamatsu ${ }^{4}$, Takaki Imai ${ }^{6}$, Nobuya Harada ${ }^{4}$, Yu Kudo $^{4}$, Naoto Shiba $^{7}$ \\ ${ }^{1}$ Department of Physical Therapy, Kyushu Nutrition Welfare University, Kitakyushu, Japan \\ ${ }^{2}$ Department of Orthopedic Surgery, Kurume University Medical Center, Kurume, Japan \\ ${ }^{3}$ Department of Orthopedic Surgery, Tahara Orthopedic Clinic, Kitakyushu, Japan \\ ${ }^{4}$ Kurume University School of Medicine Graduate School, Kurume, Japan \\ ${ }^{5}$ Department of Physical Therapy, Technical School of Medical and Welfare Ryokuseikan, Tosu, Japan \\ ${ }^{6}$ Department of Rehabilitation, Keishinkai Hospital, Tosu, Japan \\ ${ }^{7}$ Department of Orthopedic Surgery, Kurume University, Kurume, Japan
}

Background: Several systematic reviews have reported on the conservative treatment of full-thickness rotator cuff tears; however, clinical results of this treatment still remain determined.

Methods: PubMed, Cochrane Library, PEDro, and CINAHL databases were systematically searched for randomized clinical trials and observational studies. Two independent researchers reviewed a total of 2,981 articles, 28 of which met the criteria for inclusion in the study. Clinical outcome measures included Constant score, visual analog scale score for pain, range of motion, and short-form 36 questionnaires. The meta-analysis used a linear mixed model weighted with the variance of the estimate.

Results: The meta-analysis showed a significant improvement after surgery. Pain score is $26.2 \mathrm{~mm}$ (1 month) to $26.4 \mathrm{~mm}$ ( $3 \mathrm{months}$ ), and $24.8 \mathrm{~mm}$ (12 months) $(\mathrm{P}<0.05)$; active abduction: $153.2^{\circ}$ (2 months), $159.0^{\circ}$ (6 months), $168.1^{\circ}$ (12 months) ( $\left.<<0.05\right)$; Constant score: 67.8 points ( 2 months) to 77.2 points ( 12 months) $(\mathrm{P}<0.05)$; short-form 36 questionnaires "vitality" section: 57.0 points $(6$ months $)$ to 70.0 points (12 months) $(\mathrm{P}<0.05)$.

Conclusions: Our data confirmed the effectiveness of conservative treatment in patients with full-thickness rotator cuff tears 12 months post-intervention. The results suggest that conservative treatment for patients with full-thickness rotator cuff tears should be the first line of treatment before considering surgery.

Keywords: Shoulder; Rotator cuff injuries; Conservative management

\section{INTRODUCTION}

Rotator cuff tear is a common condition in middle-aged and elderly patients. Yamamoto et al. [1] investigated the prevalence of rota- tor cuff tear in 683 patients and reported that $20 \%-50 \%$ of individuals aged $>60$ years had such an injury. Moreover, Yamaguchi et al. [2] investigated 588 patients and found that 376 (63.9\%) had rotator cuff tear. Symptoms of rotator cuff tear include pain, weak-

Received: January 25, $2020 \quad$ Revised: March 21, $2020 \quad$ Accepted: March 24, 2020

Correspondence to: Masaki Karasuyama

Department of Physical Therapy, Kyushu Nutrition Welfare University, 1-5-1 Kuzuhara Takamatsu, Kitakyushu, Fukuoka 800-0298, Japan

Tel: +81-93-471-7912, Fax: +81-93-473-4161, E-mail: arukanjero@yahoo.co.jp, ORCID: https://orcid.org/0000-0002-4964-9033

Financial support: None.

Conflict of interest: None.

Copyright@ 2020 Korean Shoulder and Elbow Society. All Rights Reserved.

This is an Open Access article distributed under the terms of the Creative Commons Attribution Non-Commercial License (http://creativecommons.org/licenses/by-nc/4.0/) which permits unrestricted non-commercial use, distribution, and reproduction in any medium, provided the original work is properly cited. 
ness, and limitation of motion. In these patients, conservative treatment is primarily chosen and includes nonsteroidal anti-inflammatory drug administration, steroid injection, hyaluronic acid injection, physical therapy, and exercise therapy. Previous studies reported acceptable results in patients with rotator cuff tear treated with combinations of rehabilitation and local corticosteroid injections [3].

Systematic reviews on the conservative treatment of full-thickness rotator cuff tear (FT-RCT) have been reported [4]; however, previous reports have not provided sufficient knowledge on conservative treatment due to a paucity of high-quality studies. These results prompted us to conduct a systematic review of the conservative treatment of FT-RCT, including observational studies. Therefore, the purpose of the present study was to perform meta-analysis of clinical outcomes of patients with FT-RCT who received conservative treatment.

\section{METHODS}

We systematically searched the PubMed, Cochrane Library, PEDro, and CINAHL databases for studies conducted between January 1992 and July 2017, with the search terms rotator cuff, rotator cuff tear, subacromial impingement syndrome, rehabilitation, physiotherapy, physical therapy, exercise, conservative, and nonoperative. The references of the selected studies were also reviewed, when applicable, to identify additional studies. The inclusion criteria were as follows: randomized clinical trial; observational study; study investigating full thickness, massive, or inoperable rotator cuff tear; study explicitly mentioning that the treatment group received conservative treatment for this condition; and study reporting one or more of the outcome measures.

A meta-analysis was performed to estimate the clinical outcomes of conservative treatment in patients with FT-RCT. Case reports were excluded from the analysis. For each included article, synthesis began by pooling all reported outcomes gathered at all reported time points. The mean and standard deviation of the continuous results (i.e., Constant score and pain) were extracted from each study according to the follow-up period. When means or standard deviations were not reported in an article, they were calculated using the available information, if possible. Outcomes reported by two or more studies were pooled in a meta-analysis. Constant score, pain (visual analog scale [VAS]), range of motion (ROM) (active flexion/active abduction), and short-form 36 questionnaires (SF-36) results were the selected outcomes measured because they were reported as the mean and standard deviation of effect indicators in the included studies. The reasons for the outcomes that could not be analyzed were as follows: only one article was used as an effect indicator; articles used as effect indicators with unknown average or standard deviations, and a differing measurement method from other articles.

Analysis was performed using JMP ver. 13 (SAS Institute Inc., Cary, NC, USA). The meta-analysis used a linear mixed model [5] weighted with the variance of the estimate. A P-value less than 0.05 was considered statistically significant.

\section{RESULTS}

\section{Study Selection}

The initial database search yielded 2,981 abstracts. After removal of duplicates, 2,881 articles remained for review, of which 28 met the criteria for inclusion in the study. Of all included studies, six were randomized clinical trials, four were cohort studies, 15 were case series, and three were case reports (Fig. 1).

\section{Characteristics of Studies}

A total of 1,824 patients was included in the 28 investigations reviewed. The average age of patients who participated in these investigations was 62.9 years ( $44-83$ years). The intervention period of the conservative treatment in these investigations ranged from 3 weeks to 2 years. The mean final outcome measurements were performed at a minimum of 2 months to a maximum of 7.6 years (Table 1) [3,6-32].

The outcome measures used in studies were ROM (15 trials) [6,8,11-14,16,18-20,24-26,31,32], Constant score (13 trials) [3,9$11,14,16,18-22,24,30]$, pain (10 trials) $[3,6,8-10,12,16,18,19,22]$, strength (10 trials) [7,8,13,14,16,19-21,25,32], American Shoulder and Elbow Surgeons score (seven trials) [7,15,19,20,29-31], SF-36 score (four trials) [19,20,23,27], Western Ontario Rotator Cuff Index (three trials) $[7,8,15]$, Simple Shoulder Test score (three trials) $[9,14,27]$, University of California at Los Angeles Shoulder Score (two trials) [26,31], Japanese Orthopedic Association Score (two trials) $[17,28]$, satisfaction (two trials) $[19,26]$, shoulder rating questionnaire (one trial) [25], disability (one trial) [9], impairment (one trial) [22], Modified Wolfgang's Criteria (one trial) [32], Disabilities of the Arm, Shoulder, and Hand score (one trial) [7], Quick Disabilities of the Arm, Shoulder, and Hand score (one trial) [6], Oxford Shoulder Disability Questionnaire score (one trial) [23], SF-12 score (one trial) [15], Single Assessment Numeric Evaluation score (one trial) [15], shoulder activity scale score (one trial) [15], night pain (one trial) [18], EuroQol questionnaire score (one trial) [16], Rotator Cuff Quality of Life Index (one trial) [13], Global Rating of Change (one trial) [6], Dutch Simple Shoulder Test score (one trial) [9], and shoulder functional status (one trial) [3]. 


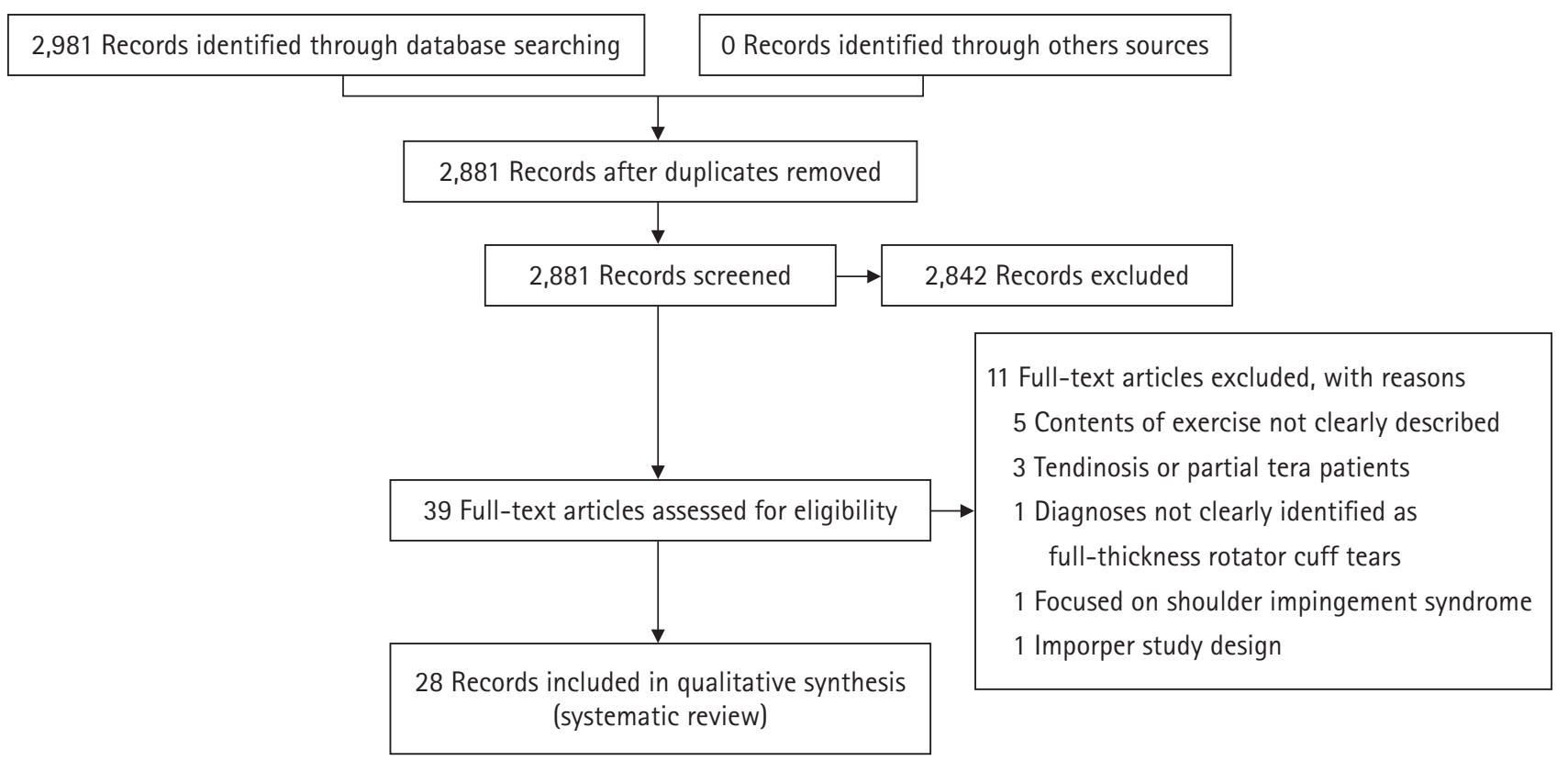

Fig. 1. Flowchart of the articles included in the systematic review.

\section{Meta-Analysis}

The analysis showed change in constant score, pain (VAS), ROM (active flexion/active abduction), and SF-36 1 year after the intervention from baseline. For the constant score, six studies $[9,16,19,20,22,24]$ involving 174 shoulders were analyzed. The Constant score was 54.3 points (baseline), 67.8 points ( 2 months), 73.1 points ( 3 months), 78.0 points ( 6 months), and 77.2 points (12 months), and trajectories over time showed a significant difference $(\mathrm{P}<0.05)$. Constant score at $2-12$ months after intervention was significantly higher than that at baseline $(\mathrm{P}<0.05)$ (Fig. 2A).

For the pain variable (VAS), six studies $[3,8,9,16,19,22]$ involving 209 shoulders were included. The change in pain (VAS) was from $59.8 \mathrm{~mm}$ (baseline) to $26.2 \mathrm{~mm}$ (1 month), $35.1 \mathrm{~mm}$ (2 months), $26.4 \mathrm{~mm}$ (3 months), $30.4 \mathrm{~mm}$ (6 months), and $24.8 \mathrm{~mm}$ (12 months), and trajectories over time showed a significant difference $(\mathrm{P}<0.05)$. Pain $(\mathrm{VAS})$ at $1-12$ months after intervention was significantly lower than that at baseline $(\mathrm{P}<0.05)$ (Fig. 2B).

For the ROM, five studies $[8,16,19,20,24]$ involving 144 shoulders were included. The ROM (active flexion/active abduction) changed from $135.7^{\circ} / 122.5^{\circ}$ (baseline) to $159.5^{\circ} / 153.2^{\circ}$ ( 2 months), $161.7^{\circ} / 156.4^{\circ}$ (3 months), $160.0^{\circ} / 159.0^{\circ}$ (6 months), and $171.9^{\circ} / 168.1^{\circ}$ (12 months) (Fig. 2C). The transition of active flexion trajectories over time showed no significant difference, but active abduction showed a significant difference $(\mathrm{P}<0.05)$. Active abduction at 2, 6, and 12 months after intervention was significantly higher than that at baseline $(\mathrm{P}<0.05)$.

For the SF-36, two studies $[19,20]$ involving 71 shoulders were included. In SF-36, the mean value of "vitality" showed a significant improvement at 6 and 12 months after intervention (all $\mathrm{P}<0.05$ ). There was no significant difference in the other seven subscales because of missing data; however, the mean values of these scales tended to improve at 6 months after intervention (Fig. 3).

\section{DISCUSSION}

Several systematic reviews have reported conservative treatment approaches for FT-RCT. Seida et al. [33] reported a systematic review of conservative and surgical treatments. The review indicated limited data needed to reach clear conclusions for most of the interventions investigated. Recently, Piper et al. [34] used meta-analysis to compare conservative and surgical treatments of FT-RCTs. They reported a statistically significant improvement in clinical outcomes of surgical treatment compared to conservative treatment for patients with rotator cuff tear. However, clinical results from conservative treatment are limited. Therefore, the present meta-analysis focused on the effectiveness of conservative management in patients with FT-RCT by including randomized trials and observational studies. Our data showed that, in patients with FT-RCT who underwent conservative management, pain (VAS), ROM (active flexion/abduction), and Constant score improved at 3 months after treatment and SF-36 improved at 6 months after treatment.

In this meta-analysis, we found that treatment response in terms of Constant score followed a pattern of rapid improvement in the 


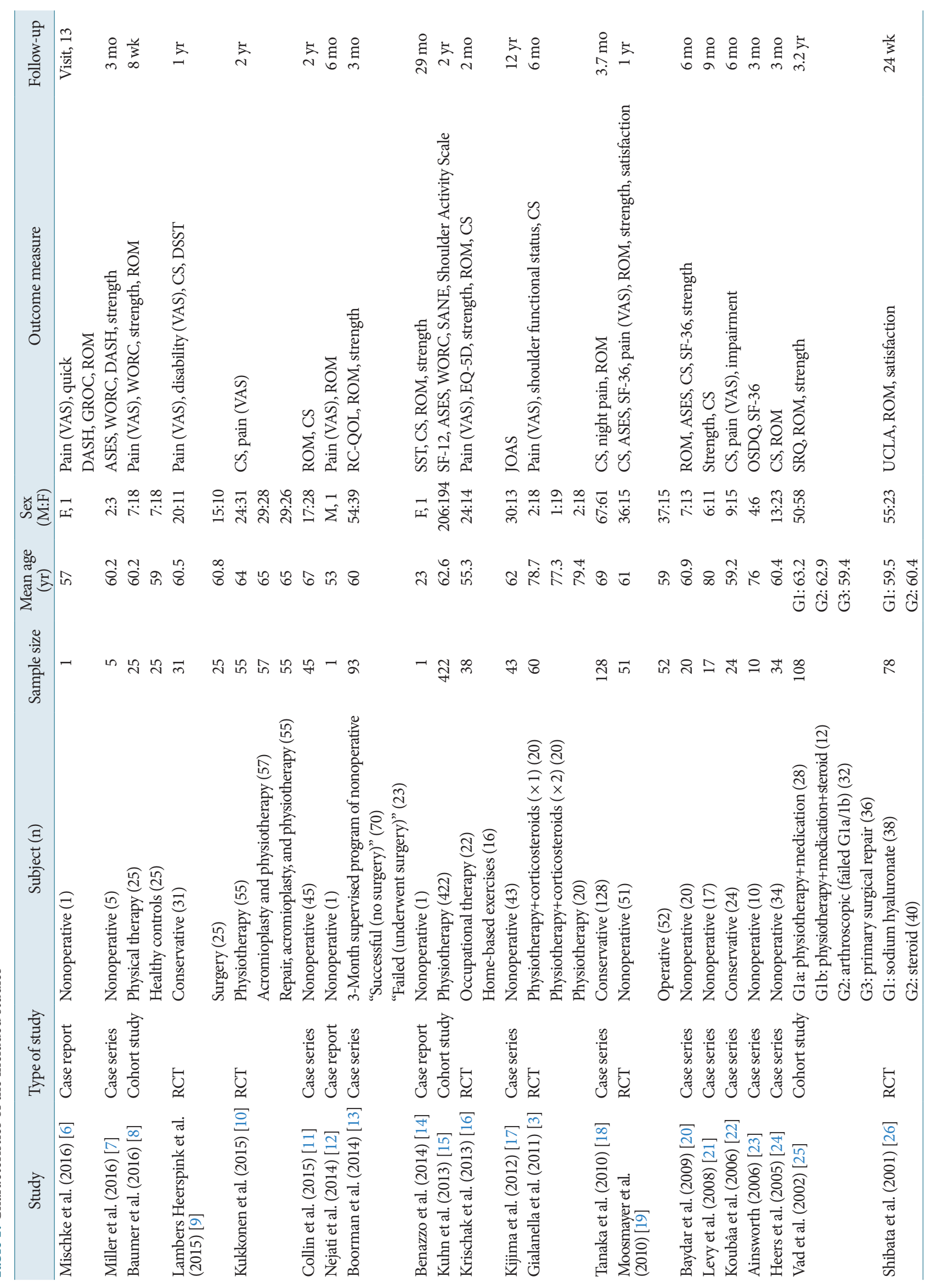




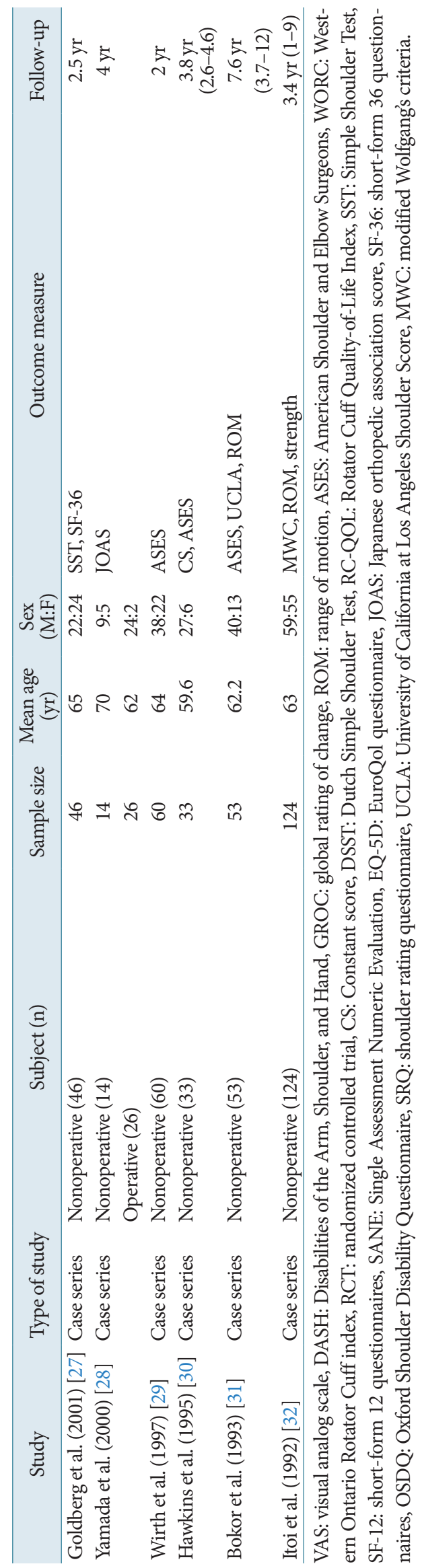

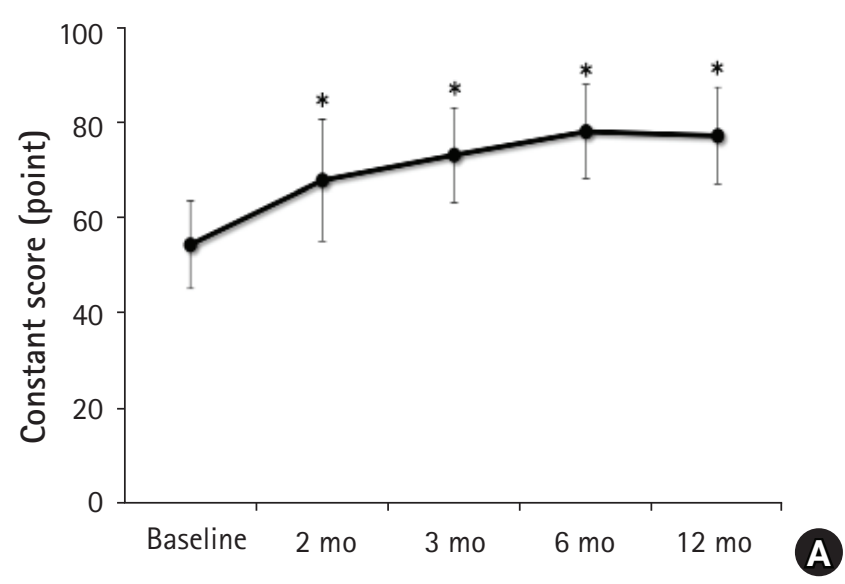
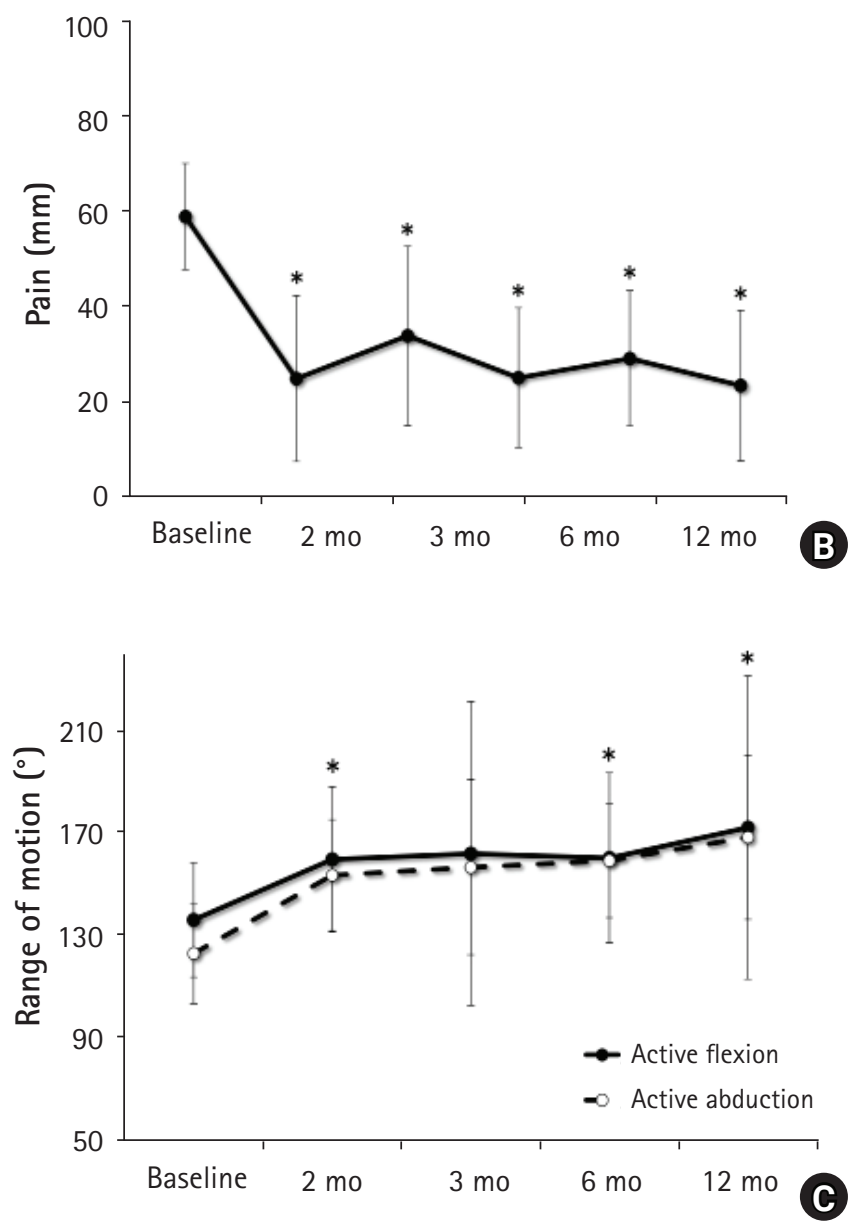

Fig. 2. Plot showing the Constant score (A), pain (B), and active flexion/active abduction (C) for the conservative management group. Whiskers indicate $95 \%$ confidence interval. ${ }^{\star} \mathrm{P}<0.05$.

first 2 months after intervention and then recovery plateaus. Baydar et al. [20] showed that the Constant score significantly improved 6 months after conservative treatment in patients with FTRCT. Moosmayer et al. [19] compared patient outcomes after sur- 


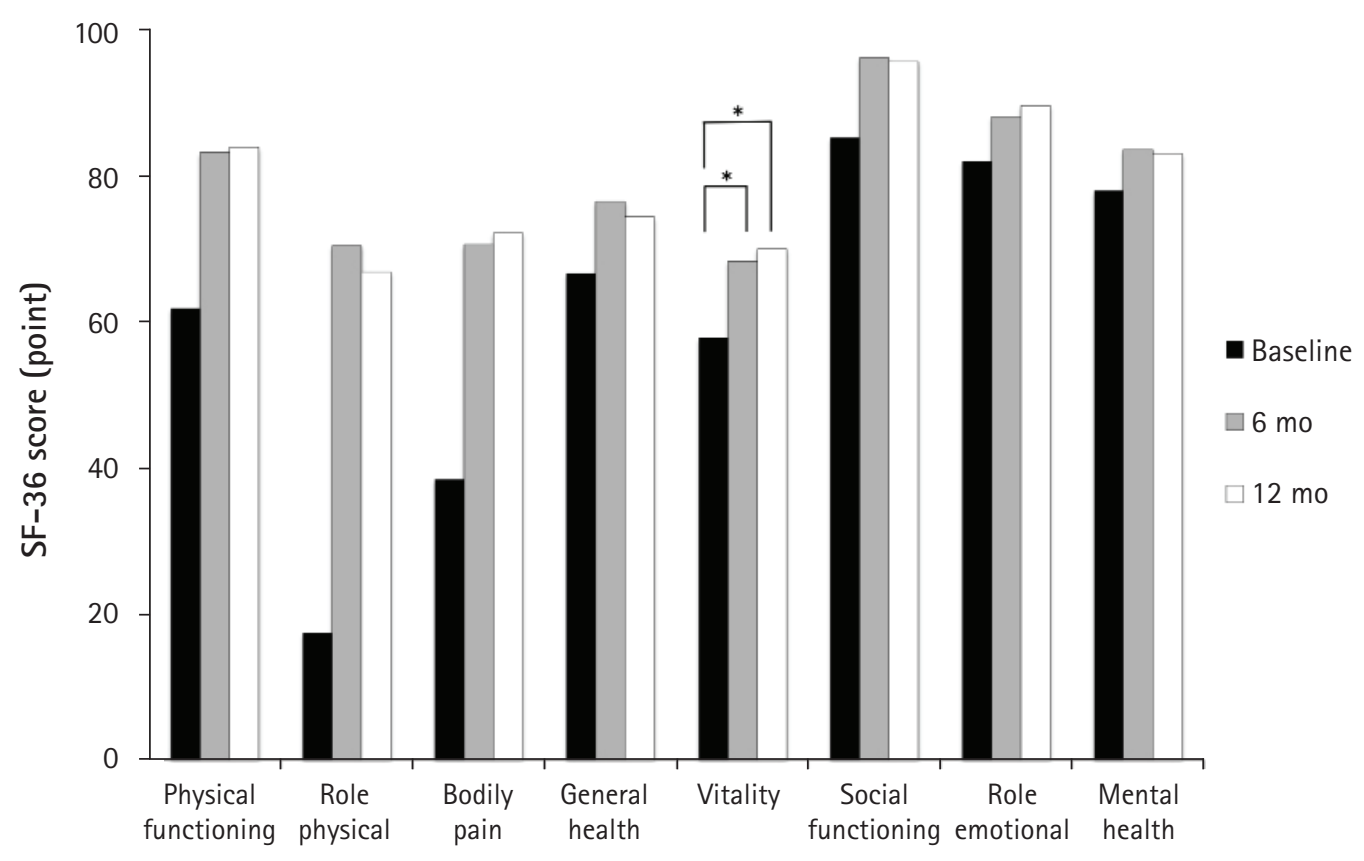

Fig. 3. Graph showing the short-form 36 questionnaires (SF-36) score for the conservative management group. ${ }^{\star} \mathrm{P}<0.05$.

gical or conservative treatment of FT-RCTs. Based on their results, 1 year after treatment, there was an improvement in the mean Constant score in both groups. The results of these studies suggest that conservative treatment produces satisfactory outcomes in the short and medium term. In this study, the Constant score significantly improved 2 months after treatment. Therefore, these results suggest that conservative treatment leads to significant improvement in functional outcomes in the first 2 months after therapy, after which the recovery plateaus.

Moosmayer et al. [19] showed that the pain score improved at 1-year follow-up in patients with FT-RCT who received conservative treatment. Similarly, Koubâa et al. [22] reported that patients with FT-RCT who received conservative treatment had improved pain scores. We showed that patients with FT-RCT demonstrated improved pain with conservative management. Altogether, the results of this review suggest the significance of improved pain within 2 months.

Several studies have reported that short-term and medium-term conservative treatment has a positive effect on ROM in patients with FT-RCT. Baumer et al. [8] reported that ROM in 25 patients with FT-RCT significantly improved 2 months after conservative treatment. Baydar et al. [20] and Moosmayer et al. [19] reported that ROM significantly improved 6 months after conservative treatment. In the present study, ROM significantly improved 2, 6, and 12 months after treatment. Thus, these results are consistent with previous studies on the importance of conservative treatment to improve ROM in patients with FT-RCT.

The SF-36 was developed in 1997, and consisted of eight independent items of general health, physical functioning, role physical, bodily pain, vitality, social functioning, role emotional, and mental health. The SF-36 scale has been widely used to evaluate patient quality of life, including both physical and mental health. The scales and summary components ranged from 0 to 100 , of which higher values denote better functioning and fewer limitations. The present study evaluated this patient-based assessment score in patients with FT-RCT who underwent conservative treatment. As a result, "vitality," which was related to mental health, significantly improved at 6 and 12 months after treatment. Due to the lack of data, we were unable to analyze the other factors, but we indicated the effectiveness of conservative treatment in terms of the patient-based scale.

There were several limitations in this study. First, various types of FT-RCT were included (e.g., isolated supraspinatus tear and two or three tendon tears). Second, there was a lack of uniformity in the treatment modalities among the studies evaluated. Third, this study did not perform a subgroup analysis of patients with FTRCT whose conservative treatment failed. Fourth, the parameters that indicated positive results of conservative treatment were limited. Therefore, future studies are warranted to address these issues.

This study showed that, for patients with FT-RCT who under- 
went conservative management, pain, ROM, and Constant score improved at 3 months after treatment and SF-36 improved at 6 months after treatment. Our data confirmed the effectiveness of conservative treatment in patients with FT-RCT within 12 months postintervention.

Our data confirmed acceptable results for conservative treatment in patients with FT-RCTs. Pain, ROM (active abduction), and Constant score improved 3 months after treatment, and the "vitality" score of the SF-36 improved 6 months after treatment. We also confirmed that these effects continued for 1 year after treatment. Therefore, these results suggest that conservative treatment for patients with FT-RCTs should be the first step before considering surgery.

\section{ORCID}

\section{Masaki Karasuyama}

https://orcid.org/0000-0002-4964-9033

\section{REFERENCES}

1. Yamamoto A, Takagishi K, Osawa T, et al. Prevalence and risk factors of a rotator cuff tear in the general population. J Shoulder Elbow Surg 2010;19:116-20.

2. Yamaguchi K, Ditsios K, Middleton WD, Hildebolt CF, Galatz LM, Teefey SA. The demographic and morphological features of rotator cuff disease: a comparison of asymptomatic and symptomatic shoulders. J Bone Joint Surg Am 2006;88:1699-704.

3. Gialanella B, Prometti P. Effects of corticosteroids injection in rotator cuff tears. Pain Med 2011;12:1559-65.

4. Downie BK, Miller BS. Treatment of rotator cuff tears in older individuals: a systematic review. J Shoulder Elbow Surg 2012; 21:1255-61.

5. Sutton AJ, Abrams KR, Jones DR, Sheldon TA, Song F. Methods for meta-analysis in medical research. Chichester: John Wiley and Sons; 2000.

6. Mischke JJ, Emerson Kavchak AJ, Courtney CA. Effect of sternoclavicular joint mobilization on pain and function in a patient with massive supraspinatus tear. Physiother Theory Pract 2016; $32: 153-8$

7. Miller RM, Popchak A, Vyas D, et al. Effects of exercise therapy for the treatment of symptomatic full-thickness supraspinatus tears on in vivo glenohumeral kinematics. J Shoulder Elbow Surg 2016;25:641-9.

8. Baumer TG, Chan D, Mende V, et al. Effects of rotator cuff pathology and physical therapy on in vivo shoulder motion and clinical outcomes in patients with a symptomatic full-thickness rotator cuff tear. Orthop J Sports Med 2016;4:2325967116666506.
9. Lambers Heerspink FO, van Raay JJ, Koorevaar RC, et al. Comparing surgical repair with conservative treatment for degenerative rotator cuff tears: a randomized controlled trial. J Shoulder Elbow Surg 2015;24:1274-81.

10. Kukkonen J, Joukainen A, Lehtinen J, et al. Treatment of nontraumatic rotator cuff tears: a randomized controlled trial with two years of clinical and imaging follow-up. J Bone Joint Surg Am 2015;97:1729-37.

11. Collin PG, Gain S, Nguyen Huu F, Lädermann A. Is rehabilitation effective in massive rotator cuff tears. Orthop Traumatol Surg Res 2015;101(4 Suppl):S203-5.

12. Nejati P, Akbari F. Exercise therapy for total tear of rotator cuff: a case report. Asian J Sports Med 2014;5:67-70.

13. Boorman RS, More KD, Hollinshead RM, et al. The rotator cuff quality-of-life index predicts the outcome of nonoperative treatment of patients with a chronic rotator cuff tear. J Bone Joint Surg Am 2014;96:1883-8.

14. Benazzo F, Marullo M, Pietrobono L. Supraspinatus rupture at the musculotendinous junction in a young woman: version 2. J Orthop Traumatol 2014;15:231-4.

15. Kuhn JE, Dunn WR, Sanders R, et al. Effectiveness of physical therapy in treating atraumatic full-thickness rotator cuff tears: a multicenter prospective cohort study. J Shoulder Elbow Surg 2013;22:1371-9.

16. Krischak G, Gebhard F, Reichel H, et al. A prospective randomized controlled trial comparing occupational therapy with home-based exercises in conservative treatment of rotator cuff tears. J Shoulder Elbow Surg 2013;22:1173-9.

17. Kijima H, Minagawa H, Nishi T, Kikuchi K, Shimada Y. Longterm follow-up of cases of rotator cuff tear treated conservatively. J Shoulder Elbow Surg 2012;21:491-4.

18. Tanaka M, Itoi E, Sato K, et al. Factors related to successful outcome of conservative treatment for rotator cuff tears. Ups J Med Sci 2010;115:193-200.

19. Moosmayer S, Lund G, Seljom U, et al. Comparison between surgery and physiotherapy in the treatment of small and medium-sized tears of the rotator cuff: a randomised controlled study of 103 patients with one-year follow-up. J Bone Joint Surg Br 2010;92:83-91.

20. Baydar M, Akalin E, El O, et al. The efficacy of conservative treatment in patients with full-thickness rotator cuff tears. Rheumatol Int 2009;29:623-8.

21. Levy O, Mullett H, Roberts S, Copeland S. The role of anterior deltoid reeducation in patients with massive irreparable degenerative rotator cuff tears. J Shoulder Elbow Surg 2008;17:863-70.

22. Koubâa S, Ben Salah FZ, Lebib S, Miri I, Ghorbel S, Dziri C. Conservative management of full-thickness rotator cuff tears: a 
prospective study of 24 patients. Ann Readapt Med Phys 2006; 49:62-7.

23. Ainsworth R. Physiotherapy rehabilitation in patients with massive, irreparable rotator cuff tears. Musculoskeletal Care 2006; 4:140-51.

24. Heers G, Anders S, Werther M, Lerch K, Hedtmann A, Grifka J. Efficacy of home exercises for symptomatic rotator cuff tears in correlation to the size of the defect. Sportverletz Sportschaden 2005;19:22-7.

25. Vad VB, Warren RF, Altchek DW, O'Brien SJ, Rose HA, Wickiewicz TL. Negative prognostic factors in managing massive rotator cuff tears. Clin J Sport Med 2002;12:151-7.

26. Shibata Y, Midorikawa K, Emoto G, Naito M. Clinical evaluation of sodium hyaluronate for the treatment of patients with rotator cuff tear. J Shoulder Elbow Surg 2001;10:209-16.

27. Goldberg BA, Nowinski RJ, Matsen FA 3rd. Outcome of nonoperative management of full-thickness rotator cuff tears. Clin Orthop Relat Res 2001;(382):99-107.

28. Yamada N, Hamada K, Nakajima T, Kobayashi K, Fukuda H.
Comparison of conservative and operative treatments of massive rotator cuff tears. Tokai J Exp Clin Med 2000;25:151-63.

29. Wirth MA, Basamania C, Rockwood CA Jr. Nonoperative management of full-thickness tears of the rotator cuff. Orthop Clin North Am 1997;28:59-67.

30. Hawkins RH, Dunlop R. Nonoperative treatment of rotator cuff tears. Clin Orthop Relat Res 1995;(321):178-88.

31. Bokor DJ, Hawkins RJ, Huckell GH, Angelo RL, Schickendantz MS. Results of nonoperative management of full-thickness tears of the rotator cuff. Clin Orthop Relat Res 1993;(294):103-10.

32. Itoi E, Tabata S. Conservative treatment of rotator cuff tears. Clin Orthop Relat Res 1992;(275):165-73.

33. Seida JC, LeBlanc C, Schouten JR, et al. Systematic review: nonoperative and operative treatments for rotator cuff tears. Ann Intern Med 2010;153:246-55.

34. Piper CC, Hughes AJ, Ma Y, Wang H, Neviaser AS. Operative versus nonoperative treatment for the management of full-thickness rotator cuff tears: a systematic review and meta-analysis. J Shoulder Elbow Surg 2018;27:572-6. 